\title{
Découverte de Centaurea hyalolepis Boiss. (Asteraceae) dans la région de Djelfa (Algérie)
}

\author{
Hanène Zater ${ }^{1,3}$, Khellaf Rebbas ${ }^{2}$, Sabrina Bicha ${ }^{3}$ et Sara Ouissem Bensaid ${ }^{3}$ \\ ${ }^{1}$ Département des sciences de la matière, Faculté des sciences exactes et sciences de la nature et de la vie, \\ Université Oum El Bouaghi, Algérie. \\ ${ }^{2}$ Département des sciences de la nature et de la vie, faculté des sciences, université Mohamed Boudiaf de M'Sila, \\ Algérie. Laboratoire d'Agro-Biotechnologie et de nutrition en zones arides et semi arides/ Equipe de gestion des \\ ressources naturelles et environnement. Université Ibn Khaldoun, Tiaret, Algérie. \\ ${ }^{3}$ Unité de valorisation des ressources naturelles, des molécules bioactives et des analyses physico-chimiques et \\ biologiques, département de chimie, faculté des sciences exactes, université des frères Mentouri, Constantine, Algérie.
}

\section{Correspondencia}

K. Rebbas

e-mail: khellaf.rebbas@univ-msila.dz

Recibido: 6 noviembre 2018

Aceptado: 2 septiembre 2019

Publicado on-line: 20 septiembre 2019

Editado por: B. Cabezudo
Discovery of Centaurea hyalolepis Boiss. (Asteraceae) in the region of Djelfa (Algeria)

Mots clés: Asteraceae, Centaurea hyalolepis, découverte, Djelfa.

Key words: Asteraceae, Centaurea hyalolepis, discovery, Djelfa.
Le genre Centaurea s.l. comporte environ 600 espèces réparties dans le monde (Bremer 1994; Hellwig 2004; Negaresh \& Rahiminejad 2014; Oreizi et al. 2017; Negaresh, 2018). De point de vue taxonomique, ce genre est jugé difficile en raison de la grande variabilité morphologique et caryologique et des confusions nomenclaturales (Bremer 1994; Wagenitz \& Hellwig 1996; Hellwig 2004; Garcia-Jacas et al., 2006; Susanna \&
Garcia-Jacas, 2009; Uysal et al., 2009; Devesa et al., 2012; Negaresh \& Rahiminejad 2014, 2015).

La famille des Asteraceae en Algérie est la plus importante et elle comprend 408 espèces réparties en 109 genres. Le genre Centaurea comprend 46 espèces (Quézel et Santa, 1962-1963).

En Afrique du nord, Centaurea hyalolepis Boiss. (Centaurea pallescens var. hyalolepis (Boiss.) Maire; Calcitrapa hyalolepis (Boiss.) Holub) n'est

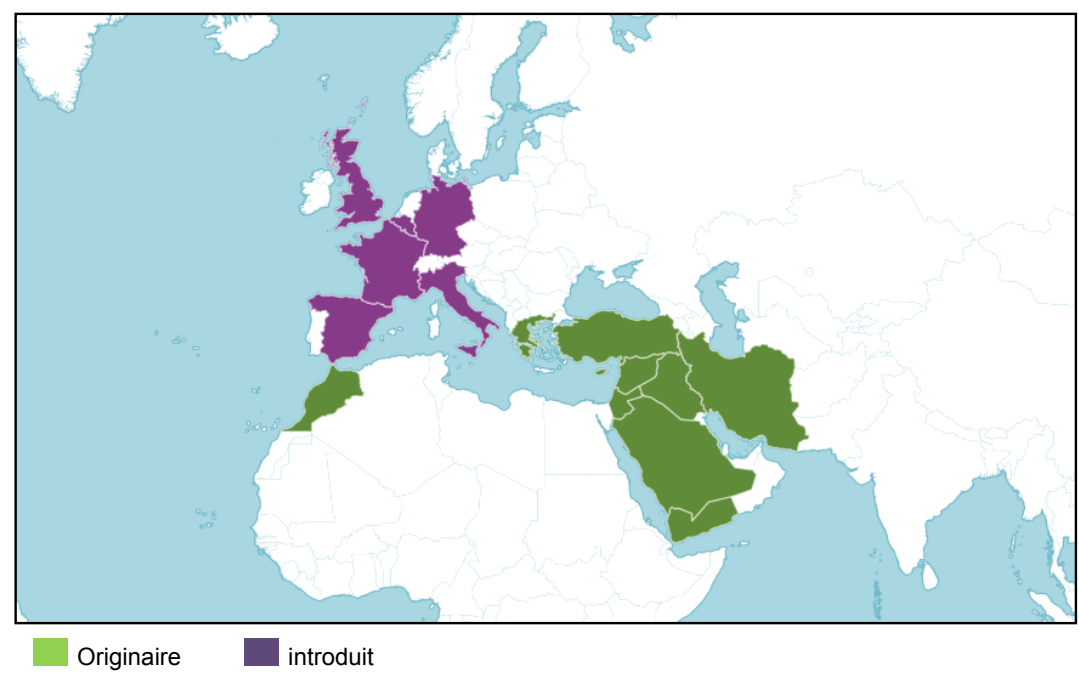

Figure 1: Répartition géographique de Centaurea hyalolepis Boiss. dans le bassin méditerranéen (Kew Science - Royal Botanic Gardens, 2018).

Figure 1: Geographic distribution of Centaurea hyalolepis Boiss. in the Mediterranean basin (Kew Science - Royal Botanic Gardens, 2018). 


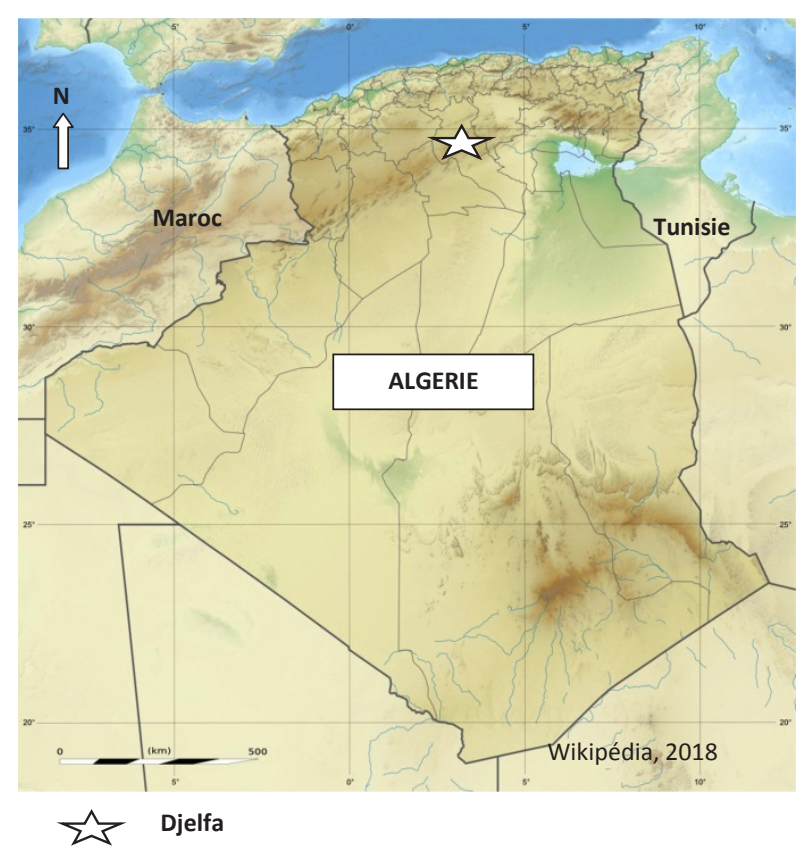

Figure 2: Localisation géographique de la station d'observation de Centaurea hyalolepis Boiss. en Algérie.

Figure 2: Geographical location of Centaurea hyalolepis Boiss observation station in Algeria.

pas citée en Algérie selon ces différents travaux (index de Dobignard \& Chatelain, 2010-2013, flore ou catalogue d'Algérie (Pomel, 1874; Battandier, 1888-1890; Battandier \& Trabut, 1905; Battandier, 1910; Maire, 1952-1987; Quézel \& Santa 19621963; I'Euro+Med PlantBase (Euro+Med 2006-).

C. hyalolepis est présente dans les pays suivants (Fig. 1) : Allemagne, Arabie Saoudite, Belgique, Chypre, Egypte, France, Espagne, Grande-Bretagne, Grèce, Iran, Israël, Italie, Irak, Jordanie, Liban, Maroc, Palestine, Syrie, Turquie, Yémen (Nahal, 1962; Ghaffari, 1989; Della, 1992; Crespo, 1992; Alziar, 1995; Euro+Med 2006; Garcia-Jacas et al., 2006; Green \& Gradner, 2010; Grace, 2010; Arnelas \& Devesa, 2011; Mosleh et al., 2013; Tison et al., 2014; Sayar et al., 2015; Awaja, 2015; Farouji \& Khodayari, 2016; Lazzeri, 2016; Dardona, 2016; Kew Science, 2018; CABI, 2018). Le présent travail vient faire le point sur la présence de ce nouveau spermaphyte récemment découvert dans la région de Djelfa, nord centre de l'Algérie.

\section{Lieu de la découverte et description botanique}

Plus d'une trentaine d'individus de Centaurea hyalolepis ont été observés sur les bords des routes et des champs dans la région de Djelfa (Fig. 2). Un spécimen d'herbier collecté a été déposé dans l'herbier officiel de l'école nationale supérieure agronomique d'Alger (Herbarium de

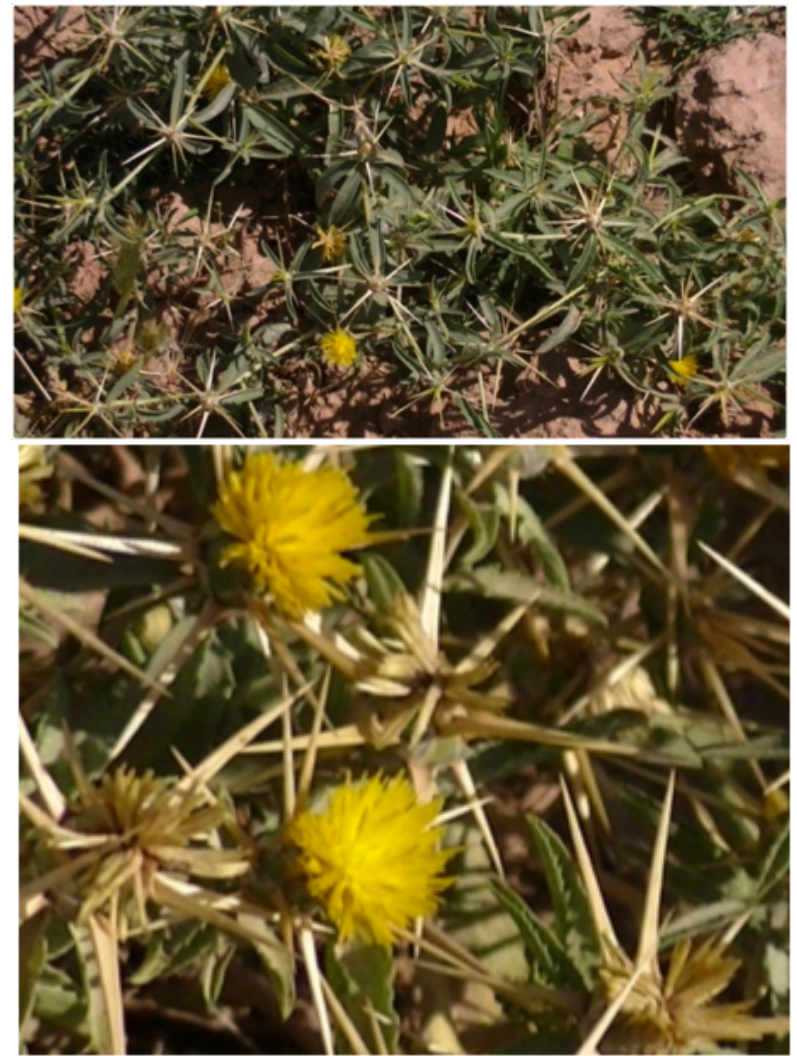

Figure 3: Illustration de Centaurea hyalolepis Boiss. (Djelfa, 20.6.2015, photos : H. Zater).

Figure 3: Illustration of Centaurea hyalolepis Boiss. (Djelfa, 20.6.2015, photos : H. Zater).

I'ENSA est inscrit dans l'index Herbariorum du New York Botanical Garden depuis 2016).

Cette Astéracée pousse sur les sols nitrifiés des zones méditerranéennes chaudes et sèches et participe à plusieurs communautés végétales rudérales appartenant principalement à la classe phytosociologique Ruderali-Secalietea Br.-BI. 1936 (Crespo, 1992).

Les couleurs des fleurs séparent facilement les deux espèces, Centaurea calcitrapa et Centaurea hyalolepis: violet ou rose violacé - rarement blanc - dans le cas de $C$. calcitrapa et jaune chez $C$. hyalolepis (Fig. 3). En outre, alors que le premier est largement répandu dans la majeure partie de la péninsule ibérique, le second n'est situé que dans l'Espagne (province d'Alicante), où il a apparemment été introduit (Crespo, 1992; Devesa Alcaraz et al., 2012).

Les fleurs chez $C$. hyalolepis possèdent des étamines avec un filament velu sur toute sa longueur et des anthères de 5-5,5 mm, jaune ; akènes de 2.5-2.7 mm de long (Devesa Alcaraz et al., 2012). 


\section{Remerciements}

Nous tenons à remercier Jean-Marc Tison (France) pour son aide dans l'identification taxonomique de cette espèce.

\section{Références}

Alziar, G. (1995). Généralités sur la flore de l'ile de Chypre. Quelques données quantitatives. Ecologia Mediterranea, XXI (1/2) : 47-52.

Arnelas, I. \& Devesa J.-A. (2011). Revisión taxonómica de Centaurea sect. Jacea (Mill.) Pers. (Asteraceae) en la Peninsula Ibérica. Acta Botanica Malacitana, 36: 33-88.

Awaja, S.M. (2015). Taxonomy and Ecology of Natural Vegetation in the Industrial Area, North Gaza Strip, Palestine. Thesis of Master in Biological, The Islamic University, Gaza, 101p.

Battandier, J. A. (1888-1890). Flore d'Algérie: Ancienne flore d'Alger transformée (Dicotylédones). Alger. 892p.

Battandier, J. A. \& Trabut, L. C. (1905). Flore analytique et synoptique de l'Algérie et de la Tunisie. Alger. 460p.

Battandier, J. A. (1910). Flore de l'Algérie: Supplément aux Phanérogames. Alger. 90p.

Bremer, K. (1994). Asteraceae: Cladistics and Classification. Timber Press, Portland, Oregon, $752 \mathrm{p}$.

CABI. (2018). Centaurea hyalolepis Boiss. Invasive Species Compendium. https://www.cabi.org/isc/ datasheet/113063

Crespo, M. B. (1992). Centaurea hyalolepis Boiss. subsp. hyalolepis (Asteraceae), a new taxon for the Iberian peninsula. Israel J. Bot. 41: 129-133.

Dardona, A.W.Y. (2016). Floristic biodiversity and phytogeographical study in few sites of Gaza strip, Palestine. Int. J Curr. Sci.,19(1): 165-182.

Della, A. (1992). Flora of a valley in the Pendakomo area. Agricultural Research Institute, Nicosia, Cyprus. Miscellaneous Report 52, 1-12.

Devesa Alcaraz, J. A., López, E., Invernón, V. R. \& López, G. (2012). Centaurea sect. calcitrapa (Heister Ex Fabr.) DC. en la Peninsula Iberica. Lagascalia, 32: 241-260.

Dobignard, A. \& Chatelain, C. (2010/2013). Index synonymique de la flore d'Afrique du Nord. Genève.

Euro+Med (2006). Euro+Med PlantBase-the information resource for Euro-Mediterranean plant diversity. Published on the Internet http://ww2.bgbm.org/ EuroPlusMed/

Farouji, A.E. \& Khodayari, H. (2016). Evaluation of vegetation types in the West Zagros (Beiranshahr region as a case study), in Lorestan Province, Iran. Biodiversitas, 17 (1): 1-10.

Garcia-Jacas, N., Uysal, T., Romashchenko K., SuárezSantiago, V.N., Ertuğrul, K. \& Susanna, A. (2006). Centaurea revisited: a molecular survey of the Jacea group. Annals of Botany, 98: 741-753.

Ghaffari, S.M. (1989). Chromosome studies in Iranian Compositae. Iran. Journ. Bot. 4(2):189-196.

Grace, A. (2010). Introductory Biogeography to Bees of the Eastern Mediterranean and Near East. Bexhill Museum. Sussex. United Kingdom. First Edition, 285p.

Green, I. \& Gradner, C. (2010). A Greentours Tour
Report. Black Irises. March 26 th - April 8 th 2010. Jordan, 33p.

Hellwig, F. H. (2004). Centaureinae (Asteraceae) in the Mediterranean - history of ecogeographical radiation. Plant Systematics and Evolution 246: 137-162.

Kew Science (Royal Botanic Gardens). (2018). Centaurea hyalolepis Boiss. Plants of the World online. http://powo.science.kew.org/taxon/urn:Isid:ipni. org:names:190681-1

Lazzeri, V. (2016). The alien vascular flora of Tuscany (Italy): update and analysis. Quad. Mus. St. Nat. Livorno, 26: 43-78.

Maire, R. (1952-1987). Flore de l'Afrique du Nord (Maroc, Algérie, Tunisie, Tripolitaine, Cyrénaïque et Sahara). Ed. Le chevalier, Paris, V. 1-16.

Mosleh, L., Asadi, F. \& Safaian, R. (2013). Investigation on the values of International Parishan Lake's plants. Int J Adv Biol Biom Res., 1(10):1263-1270.

Nahal, I. (1962). Contribution à l'étude de la végétation dans le Baer-Bassit et le Djebel Alaouite de Syrie. Tipografia «L'impronta», Firenze, 178p.

Negaresh, K. \& Rahiminejad, M. R. (2014). A contribution to the taxonomy of Centaurea sect. Cynaroides (Asteraceae, Cardueae-Centaureinae) in Iran. Phytotaxa, 158: 229-244.

Negaresh, K. \& Rahiminejad, M.R. (2015). A taxonomic revision of Centaurea sect. Microlophus (Asteraceae, Cardueae-Centaureinae) and three new records for the flora of Iran. Nordic Journal of Botany, 33: 335353.

Negaresh, K. (2018). Lectotypification of two Turkish endemic taxa of Centaurea L. (Asteraceae, Cardueae, Centaureinae). Adansonia, sér. 3, 40 (10): 135-139.

Oreizi E., Negaresh, K. \& Rahiminejad, M. R. (2017). A new name in Centaurea (Asteraceae, Cardueae) from Turkey. Candollea, 72: 319-322.

Pomel, A. (1874). Nouveaux matériaux pour la flore atlantique. 1 - Paris, Alger (I-III, 1-156).

Sayar, M.S., Han, Y., Basbag, M., Gul, I. \& Polat, T. (2015). Rangeland improvement and management studies in the southeastern Anatolia region of Turkey. Pak. J. Agri. Sci., 52(1): 9-18.

Susanna, A. \& Garcia-Jacas, N. (2009). Cardueae (Carduoideae), in Funk V.A., Susanna A., Stuessy T. F. \& Bayer R.J. (eds), Systematics, Evolution, and Biogeography of Compositae. Vienna, International Association for Plant Taxonomy, 293-313.

Tison, J.-M., Jausein, P. \& Michaud, H. (2014). Flore de la France méditerranéenne continentale. Naturalia

Publications, 1943p.

Quézel, P. \& Santa, S. (1962-1963). Nouvelle flore de l'Algérie et des régions désertiques méridionales, CNRS. Paris. T1-2.

Uysal, T., Ertuğrul, K., Susanna, A. \& Garcia-Jacas, N. (2009). New chromosome counts in the genus Centaurea (Asteraceae) from Turkey. Botanical Journal of the Linnean Society, 159: 280-286.

Wagenitz G. \& Hellwig F. H. (1996). Evolution of characters and phylogeny of the Centaureinae, in Hind D. J. N. \& Beentje H. J. (eds), Compositae: Systematics. Proceedings of the International Compositae Conference, Kew, 1994. Royal Botanic Gardens, Kew: 491-510. 